\title{
Application with the experimental results of the Roundrobin of a 3D modelling SMA based on the based on the phase transformation surface identification under proportional loading and anisothermal conditions
}

\author{
E. Gibeau ${ }^{\mathrm{a}}$, C. Lexcellent ${ }^{\mathrm{b}}$ and L. Boubakar ${ }^{\mathrm{c}}$ \\ FEMTO-St Département de Mécanique Appliquée, 24 rue de L’Epitaphe 25000 Besançon France
}

\begin{abstract}
We present a model based on the phase transformation surface of SMA. This model is described per a thermodynamical point of view. We applied this modelling on an experimental part that has been realized by P. Sittner et al. on a nickel titane wire. We had several experimental sets with we could show the performances of our model, in particular when the taking into account of the asymmetry of the yield surface and the respect of the hysteresis width.
\end{abstract}

\section{Description of the model used, based on the phase surface identification of the material}

The model used is largely inspired from the model defined by C. Lexcellent et al.in [1]. This model is based on the experimental identification of the phase transformation surface, it suggests a modelling of shape memory alloys (SMA). Within the framework of the thermodynamics of irreversible processes, the stress-induced martensite volume fraction and the self-accommodating martensite volume fraction are chosen as internal variables. The asymmetry between tension and compression behaviours is specially studied and taken into account in the modelling.

\subsection{SMA behaviour modelling basic concepts}

Through our study, we consider a representative volume element (RVE) of SMA ; in the reference free stress state and the reference temperature $\mathrm{T}_{0}$, we observe conventionally a phase called "austenite" (A) regarded as a high-temperature phase. It can be transformed in a self-accommodating martensite $\left(\mathrm{M}_{\mathrm{T}}\right)$ by pure cooling or in a stress-induced martensite $\left(\mathrm{M}_{\sigma}\right)$ under pure mechanical loading.

It is based on a free energy function of Gibbs in which we have a typical term of interaction energy:

_Free energy function (Leclercq et Lexcellent, 1996):

$\phi_{n}\left(\underline{\varepsilon}_{\alpha}, T, z_{T}, z_{\sigma}, H_{k}\right)=(1-z) \phi_{1}+z_{T} \phi_{2}+z_{\sigma} \phi_{3}+\Delta \phi$

$\alpha=1,2,3$ (1: Austenite, 2 : self-accommodating martensite, 3 : stress-induced martensite)

$z=z_{T}+z_{\sigma} \quad z, z_{T}, z_{\sigma} \in[0,1]$

_The energy of each phase is described by:

$\phi_{\alpha}=u_{0}^{\alpha}-T s_{0}^{\alpha}+\frac{1}{2 \rho}\left(\underline{\varepsilon}_{\alpha}-\underline{\varepsilon}_{\alpha}^{t r}-\underline{\varepsilon}_{\alpha}^{t h}\right): \underline{\underline{L}}:\left(\underline{\varepsilon}_{\alpha}-\underline{\varepsilon}_{\alpha}^{t r}-\underline{\varepsilon}_{\alpha}^{t h}\right)+c_{v}\left[\left(T-T_{0}\right)-T \ln \left(\frac{T}{T_{0}}\right)\right]$

aelie.gibeauduniv-fcomte.fr, bhristian.lexcellenteuniv-fcomte.fr,

lamine.boubakar@univfcomte.fr

$\Delta \phi=z(1-z) \phi_{i t}(T)+z_{T} z_{\sigma} \phi_{i t}^{m}(T) \quad$ where $\quad \phi_{i t}(T)=\bar{u}_{0}-T \bar{s}_{0}, \quad \phi_{i t}^{m}(T)=\bar{u}_{0}^{m}-T \bar{s}_{0}^{-m}$

This is an Open Access article distributed under the terms of the Creative Commons Attribution-Noncommercial License (http://creativecommons.org/licenses/by-nc/3.0/), which permits unrestricted use, distribution, and reproduction in any noncommercial medium, provided the original work is properly cited. 
Where $z_{T}$ and $z_{\sigma}$ are the internal variables and respectively the self-accommodating martensite volume fraction and the stress-induced martensite volume fraction.

_As $\rho g=\rho \phi-\underline{\sigma}: \underline{\varepsilon}$, we obtain the Gibbs energy function:

$\rho g=\rho\left(u_{0}^{1}-T s_{0}^{1}-z \pi_{0}^{f}(T)+c_{v}\left[\left(T-T_{0}\right)-T \ln \left(\frac{T}{T_{0}}\right)\right]\right)-\frac{1}{2} \underline{\sigma}: \underline{\underline{L^{-1}}}: \underline{\sigma}-z_{\sigma} \underline{\sigma}: \underline{K}_{e q}$

$-\alpha\left(T-T_{0}\right) \underline{\sigma}: \underline{1}+\rho z(1-z) \phi_{i t}+\rho z_{T} z_{\sigma} \phi_{i t}^{m}$

_We notice that our model distinguishes the elasticity parts of austenite and martensite, with

$\underline{\underline{L^{-1}}}=\left((1-z) \underline{\underline{L_{A}^{-1}}}+z \underline{\underline{L_{M}^{-1}}}\right)$

_Then we deduce the strain, the entropy and the driving forces:

$\left\{\begin{array}{l}\underline{\varepsilon}=-\rho \frac{\partial g}{\partial \underline{\sigma}}=\underline{\varepsilon}^{e}+\underline{\varepsilon}^{t r}+\underline{\varepsilon}^{t h}, \quad \underline{\varepsilon}^{e}=\underline{\underline{L^{-1}}} \underline{\underline{\sigma}}, \quad \underline{\varepsilon}^{t r}=z_{\sigma} \underline{K}_{e q}, \quad \underline{\varepsilon}^{t h}=\alpha\left(T-T_{0}\right) \underline{1} \\ s=-\frac{\partial g}{\partial T}=s_{0}^{1}-z \Delta s_{0}+\frac{\alpha}{\rho} \underline{\sigma}: \underline{1}+z(1-z) \overline{s_{0}}+z_{T} z_{\sigma} s_{0}^{m}+C_{v} \ln \left(\frac{T}{T_{0}}\right) \\ \left.\pi_{\sigma}^{f}=-\frac{\partial g}{\partial z_{\sigma}}=\frac{1}{\rho} \underline{\sigma} \underline{e q}-(1-2 z) \phi_{i t}+\frac{1}{2} \underline{\underline{\sigma}}: \underline{\underline{\left(L_{M}^{-1}\right.}}-\underline{\underline{L_{A}^{-1}}}\right) \underline{\sigma}-z_{T} \phi_{i t}^{m}+\pi_{0}^{f}(T) \\ \left.\pi_{T}^{f}=-\frac{\partial g}{\partial z_{T}}=\frac{1}{2} \underline{\sigma}: \underline{\underline{\left(L_{M}^{-1}\right.}}-\underline{\underline{L_{A}^{-1}}}\right) \underline{\sigma}-(1-2 z) \phi_{i t}-z_{\sigma} \phi_{i t}^{m}+\pi_{0}^{f}(T)\end{array}\right.$

The Clausius-Duhem inequality gives for the intrinsic dissipation the following expression:

$D_{i}=\pi_{\sigma}^{f} \dot{z}_{\sigma}+\pi_{T}^{f} \dot{z}_{T} \geq 0$

Several cases have been distinguished:

(i) Pure thermal loading: $A \leftrightarrow M_{T}$.

Here $z_{\sigma}=0$ and $D_{i}=\pi_{T}^{f} \dot{z}_{T} \geq 0$. Then:

$A \rightarrow M_{T}$ if $\dot{z}_{T} \geq 0$ i.e. $\pi_{T}^{f} \geq 0$.

$M_{T} \rightarrow A$ if $\dot{z}_{T} \leq 0$ i.e. $\pi_{T}^{f} \leq 0$.

(ii) $\quad$ Pure stress loading: $A \leftrightarrow M_{\sigma}$.

Here $z_{T}=0$ and $D_{i}=\pi_{\sigma}^{f} \dot{z}_{\sigma} \geq 0$. Then:

$A \rightarrow M_{\sigma}$ if $\dot{z}_{\sigma} \geq 0$ i.e. $\pi_{\sigma}^{f} \geq 0$.

$M_{\sigma} \rightarrow A$ if $\dot{z}_{\sigma} \leq 0$ i.e. $\pi_{\sigma}^{f} \leq 0$.

The creation of self-accommodating and/or stress-induced martensite is then signalled by the driving forces associated. During the loading / unloading process we constantly survey the driving forces $\pi_{T}^{f}$ and $\pi_{\sigma}^{f}$ respectively associated with self-accommodating martensite and the stress-induced martensite.

To model the system evolution and the kinetics, constitutive functions are assumed: $\psi_{F}^{\sigma}, \psi_{F}^{\sigma}, \psi_{F}^{\sigma}$ and $\psi_{F}^{\sigma}$. (R: reverse phase transformation martensite $\rightarrow$ austenite, F: forward phase transformation austenite $\rightarrow$ martensite). 
$\psi_{F}^{\sigma}\left(\underline{\sigma}, T, z_{\sigma}, z_{T}\right)=\pi_{\sigma}^{f}-k_{F}^{\sigma}, \psi_{F}^{T}\left(T, z_{\sigma}, z_{T}\right)=\pi_{T}^{f}-k_{F}^{T}$,
$\psi_{R}^{\sigma}\left(\underline{\sigma}, T, z_{\sigma}, z_{T}\right)=-\pi_{\sigma}^{f}+k_{R}^{\sigma}$ and $\psi_{R}^{T}\left(T, z_{\sigma}, z_{T}\right)=-\pi_{T}^{f}+k_{R}^{T}$

We can deduce from the consistency conditions $\dot{\psi}_{F}^{\sigma}=0, \dot{\psi}_{R}^{\sigma}=0, \dot{\psi}_{F}^{T}=0$ and $\dot{\psi}_{R}^{T}=0$ the expressions of the kinetics:

$$
\begin{aligned}
z_{\sigma}^{F} & =a_{\sigma}^{F}\left\langle\sigma_{e f}-\left(s_{A M} T-b_{\sigma}^{F}\right)\right\rangle & & A \rightarrow M_{\sigma} \\
z_{\sigma}^{R} & =1-a_{\sigma}^{R}\left\langle\left(s_{M A} T-b_{\sigma}^{R}\right)-\sigma_{e f}\right\rangle & & M_{\sigma} \rightarrow A \\
z_{T}^{F} & =a_{T}^{F}\left(T-b_{T}^{F}\right) & & A \rightarrow M_{T} \\
z_{T}^{R} & =a_{R}^{T}\left(T-b_{T}^{R}\right) & & M_{T} \rightarrow A
\end{aligned}
$$

\subsection{Taking into account the asymmetry between tension and compression}

An asymmetry between tension and compression has been found for material like SMA, this effect is also called SD-effect for "strength differential effect" [2], [3] and [4]. This effect appears clearly on the surface at the initiation transformation from austenite to martensite under proportional loading. Typically, our model takes into account this phase transformation yield surface. The choice of an effective stress has been used in order to guarantee the asymmetry between tension and compression:

$$
\sigma_{e q}=\bar{\sigma} \cdot f\left(y_{\sigma}\right)
$$

With respectively the second (15) and the third (16) invariant of the stress tensor

$$
\begin{aligned}
& \bar{\sigma}=\sqrt{\frac{3}{2} \operatorname{dev}(\underline{\sigma}): \operatorname{dev}(\underline{\sigma})} \\
& y_{\sigma}=\frac{27}{2} \frac{\operatorname{det}(\operatorname{dev}(\underline{\sigma}))}{\bar{\sigma}^{3}}
\end{aligned}
$$

We introduce the SD-effect in our model through a corrective function which only depends on the third invariant of the stress tensor. We can find different corrective functions in the literature, see by example [5] and [6]. We have chosen within the framework of the experimental results the function (17)

$$
f\left(y_{\sigma}\right)=\left(1-a y_{\sigma}\right)^{n}
$$

\section{Implementation of our model for the experimental Roundrobin sets provided}

Experimental sets have been performed by P. Sittner et al., in this sets we can find tensile tests, torsion test, tensile/torsion tests, heating/cooling tests etc on a NiTi wire with a diameter of $0.1 \mathrm{~mm}$. To apply our model on this different experimental sets we had first to identify our model parameters and then to give our results.

\subsection{Model parameters identification}

The parameters we used appear in the tables 1 and 2 with their description and their values for each one. These values are directly provided by the experimentations or deduced just on tensile tests.

Table 1. Model parameters provided by the experimentations.

\begin{tabular}{|l|l|l|l|}
\hline Symbol & Description & Value & unity \\
\hline
\end{tabular}




\begin{tabular}{|c|c|c|c|}
\hline$\rho$ & Volumic mass & 6450 & $\mathrm{~kg} \cdot \mathrm{m}^{-3}$ \\
\hline$E_{A}$ & Austenite Young Modulus & 53.6 & $\mathrm{GPa}$ \\
\hline$E_{M}$ & Martensite Young Modulus & 21.6 & $\mathrm{GPa}$ \\
\hline$\nu$ & Poisson coefficient & 0.33 & - \\
\hline$\gamma$ & Maximum shear strain & 0.0465 & - \\
\hline$A_{s}^{0}$ & $\begin{array}{c}\text { Reverse transformation start } \\
\text { temperatures }\end{array}$ & 311 & $\mathrm{~K}$ \\
\hline$A_{f}^{0}$ & $\begin{array}{c}\text { Reverse transformation finish } \\
\text { temperatures }\end{array}$ & 328 & $\mathrm{~K}$ \\
\hline$M_{s}^{0}$ & $\begin{array}{c}\text { Forward transformation start } \\
\text { temperatures }\end{array}$ & 295 & $\mathrm{~K}$ \\
\hline$M_{f}^{0}$ & $\begin{array}{c}\text { Forward transformation finish } \\
\text { temperatures }\end{array}$ & 235 & \\
\hline
\end{tabular}

Table 2. Intrinsic model parameters identified

\begin{tabular}{|c|c|c|c|}
\hline Symbol & Description & Value & unity \\
\hline$\Delta u^{0}$ & $\begin{array}{l}\text { Part of the initial driving force } \\
\qquad \pi_{0}^{f}(T)\end{array}$ & 10980 & $\mathrm{~J}^{\mathrm{kg}} \mathrm{g}^{-1}$ \\
\hline$\Delta s^{0}$ & $\begin{array}{l}\text { Part of the initial driving force } \\
\qquad \pi_{0}^{f}(T)\end{array}$ & 42.58 & $\mathrm{~J}^{\prime} \mathrm{kg}^{-1} \cdot \mathrm{K}^{-1}$ \\
\hline$\overline{u_{0}}$ & Part of the internal energy $\phi_{i t}(T)$ & 3266 & $\mathrm{~J} . \mathrm{kg}^{-1}$ \\
\hline$\overline{s_{0}}$ & Part of the internal energy $\phi_{i t}(T)$ & 8.2 & $\mathrm{~J}^{\prime} \mathrm{kg}^{-1} \cdot \mathrm{K}^{-1}$ \\
\hline$\overline{u_{0}^{m}}$ & Part of the internal energy $\phi_{i t}(T)$ & 1676 & $\mathrm{~J}^{\mathrm{kg}} \mathrm{g}^{-1}$ \\
\hline$\overline{s_{0}^{m}}$ & Part of the internal energy $\phi_{i t}(T)$ & 0 & $\mathrm{~J} \cdot \mathrm{kg}^{-1} \cdot \mathrm{K}^{-1}$ \\
\hline
\end{tabular}

\subsection{Results}

We will present now several results obtained with our model. We show the main curves for each experimental set which we think they are the more relevant.

\subsubsection{Tensile tests}

Under different temperatures, we compare isothermal tensile tests on a NiTi wire with our simulations (Fig. 1) 

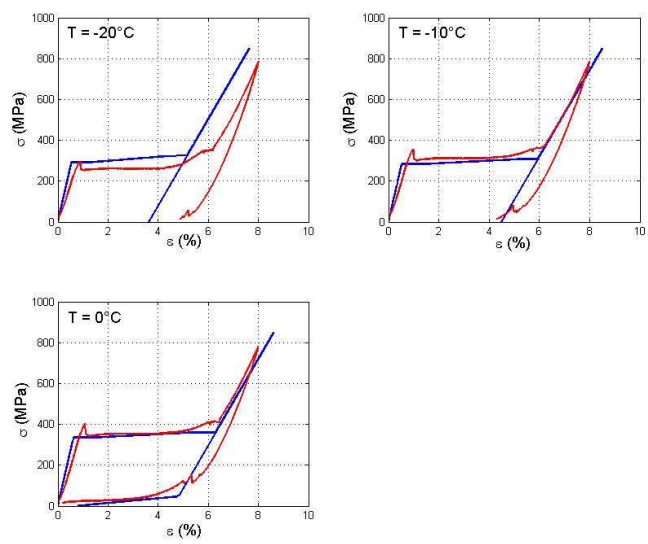
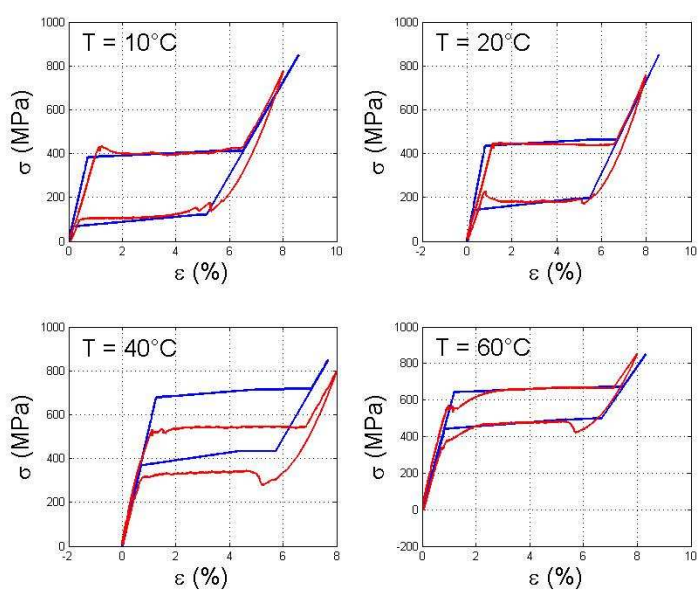

b)

Fig. 1. Comparison between the experimental tensile tests (red) and the simulations (blue) at low temperatures (a) and high temperatures (b)

\subsubsection{Torsion tests}

Isothermal torsion tests are realised under different temperatures. We observe a shape difference between the experimental and the simulation results. We can briefly explain this phenomenon by a structural approach since we don't have on a RVE and by an anisotropy of the material (Fig. 2).

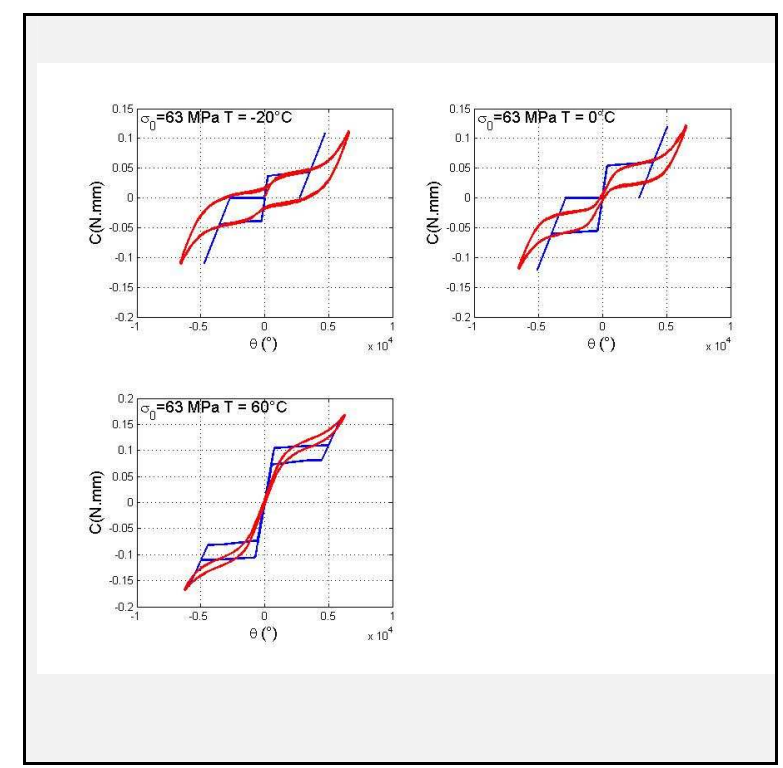

Fig. 2. Comparison between the experimental torsion tests (red) and the simulations (blue) at different temperatures

\subsubsection{Thermal tests under constant loading}

The wire has been first loaded in a first part, and then cooled/heated to create thermal cycles with different loadings (see Fig.3) 


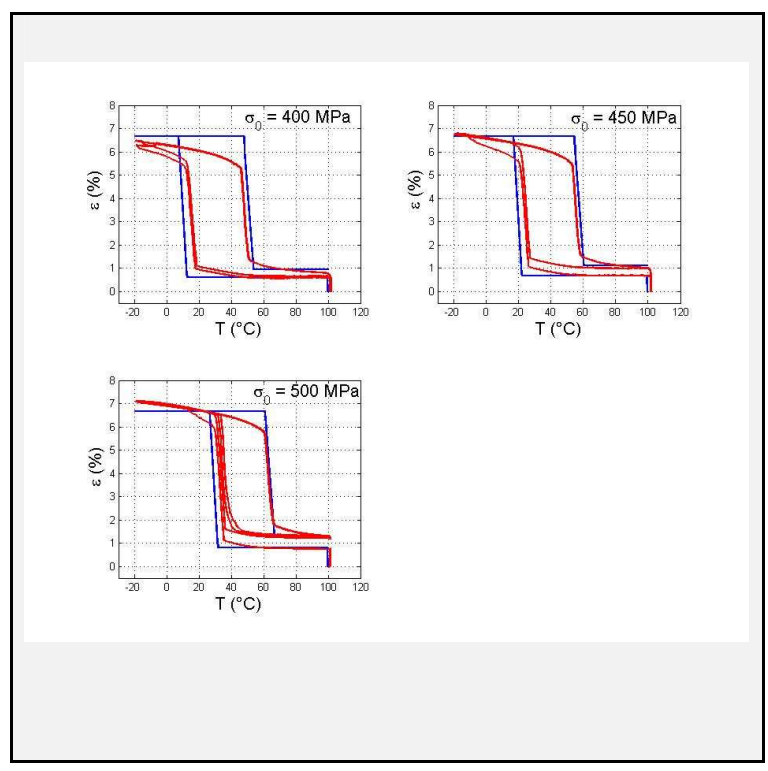

Fig. 3. Comparison between the experimental thermal tests (red) and the simulations (blue) at different temperatures

\section{Strength and weakness of this SMA model}

\section{1 strength}

The most interesting point of this modelling is the work on the yield criteria. Indeed, with a correct yield surface function ( $=$ it verifies a convexity condition) we can apprehend the different asymmetries of the material (tensile-compression) and its non-von Mises behaviour.

This SMA model takes into account the effects of the temperature. We succeeded to simulate the curves with the thermal loading and we have just identified the different parameters with tensile and torsion tests.

With this modelling, we can also capture the width of the hysteresis when the temperature changes (isothermal tensile or torsion tests). We only have one set of parameters for all the simulations. We identify on tensile and torsion tests to obtain the totality of our parameters.

\section{2 weakness}

This SMA model can't capture the slope in the torsion tests. We assume it is a structural problem with the wire. As you can see we have begun to give a theoretical explanation.

After, we have not implemented the internal loops in the program, as well we can't command in deformation. But it is just a problem of programming not modelling.

\section{Conclusion}

In conclusion, this model takes into account the phase transformation yield surface, the anisothermal transformations with constant loading or not and the proportional loadings. It distinguishes the different sorts of martensite (self-accommodating and stress-induced) and shows the evolution of each one. Also, the size of the hysteresis is respected for each type of loading.

\section{References}

[1] Ch. Lexcellent, M.L. Boubakar, Ch. Bouvet, S. Calloch, About modelling the shape memory alloy behaviour based on the phase transformation surface identification under proportional loading and anisothermal conditions Int. J. Solid. Struct. Volume, 43 (2006) ,p. 613-626

[2] W.A. Sptizig, R.J. Sober, O. Richmond, The effect of hydrostatic pressure on the deformation behavior of Maraging and HY-80 steels and its implication for plasticity theory, Metall. and Mat. Trans. Volume, 7A (1976) ,p. $1703-1710$ 
[3] T. Saburi, M. Yoshida, S. Nenno, Deformation behavior of shape memory Ti-Ni alloy crystals. Script. Metall. Volume, 18 (1984) ,p. 363-366

[4] R. Mahnken, S. Wilmanns, Simulation of asymmetric effects for shape memory alloys by decomposition of trnasformation strains. Comput. Mat. Sci. Volume, 42 (2008), p. 295-305

[5] C. Bouvet, S. Calloch, C. Lexcellent, A phenomenological model for pseudoelasticity of shape memory alloys under multi-axial proportional and non-proportional loadings. Eur J.of Mech. Sol. Volume, 23 (2004) ,p. 37-61 [6] B. Raniecki, Z. Mróz, Yield or martensitic phase transformation conditions and dissipation functions for isotropic, pressure-insensitive alloys exhibiting SD effect. Acta Mech.Volume, 195 (2008) ,p. 81-102 